\title{
SUCCESSFUL BEAM TEST OF THE HOM FREE SUPERCONDUCTING SOLEIL CAVITY PROTOTYPE AT THE ESRF
}

\author{
J. Jacob, D. Boilot, ESRF, Grenoble, France \\ S. Chel, P. Bosland, P. Brédy, CEA, Saclay, France \\ E. Chiaveri, R. Losito, CERN, Geneva, Switzerland \\ J.-M. Filhol, M.-P. Level, P. Marchand, C. Thomas-Madec, SOLEIL, Saclay, France
}

\begin{abstract}
A cryo-module housing two strongly HOM damped $352 \mathrm{MHz}$ superconducting (SC) cavity cells has been developed within the framework of the SOLEIL project design phase. In 2002, the prototype was installed in the ESRF storage ring and tested with beam in the accelerating regime with the cavities cooled down to $4.5 \mathrm{~K}$ by means of liquid helium from Dewars. Four series of tests have been carried out at the end of scheduled shutdowns. In order not to disturb the ESRF machine performance during the user mode of operation, the cavities were maintained detuned at room temperature. In this passive regime, they remained transparent to the beam with less than $100 \mathrm{~W}$ of heat deposited by the beam and evacuated by a warm helium gas flow.

Up to $170 \mathrm{~mA}$ of beam could be accelerated with a peak RF voltage of $3 \mathrm{MV}$ and a power of $360 \mathrm{~kW}$ from the SC module. This corresponds to the performance required for the first SOLEIL operation phase. The concept of effective HOM damping was validated up to the maximum ESRF intensity of $200 \mathrm{~mA}$. A few week points already identified at previous CERN tests were confirmed: high static cryogenic losses, poor cooling of one HOM coupler and too high fundamental power through the dipolar HOM couplers.
\end{abstract}

\section{INTRODUCTION}

In June 1996, a collaboration project between CEA, CNRS, CERN and ESRF was launched to develop, build and test a strongly HOM damped SC cavity for the SOLEIL project [1]. The frequency of $352.2 \mathrm{MHz}$ was chosen in order to benefit from a possible transfer of CERN technology, including the coupler design, and to open the possibility of a future implementation at the ESRF. The prototype was successfully tested at CERN in December 1999 up to $7 \mathrm{MV} / \mathrm{m}$ of accelerating gradient [2].

In December 2001, the SOLEIL module was installed in the ESRF storage ring for a period of one year, in order to validate the design with a high intensity beam [3]. For the limited test period, it was decided to feed the cryostat with liquid helium (LHe) from Dewars. Tests with the cavity at $4 \mathrm{~K}$ have been carried out following the four shutdowns of March, May, August and October 2002. After one week of pre-cooling with cold helium gas, the first machine restart days were dedicated to the tests at $4 \mathrm{~K}$. The cavity was then warmed up, kept in the ring at room temperature $(300 \mathrm{~K})$ and cooled with warm helium gas for the normal user operation, its resonance being thermally shifted off beam harmonics.

The successful operation at $300 \mathrm{~K}$ with high beam intensity - not initially foreseen in the cavity design opens the possibility of increasing the availability of SC $\mathrm{RF}$ systems if the ring can be operated with a reduced number of modules.

The cooling with LHe from Dewars was also a success: during the four series of tests, the cavity was kept stable at $4 \mathrm{~K}$ between 17 and 20 hours. With the resonance detuned from the main RF frequency, $200 \mathrm{~mA}$ could then be stored without any sign of HOM excitation. The SOLEIL cavity module was successfully operated in the accelerating regime as well: with $3 \mathrm{MV}$ of accelerating voltage, a maximum of $360 \mathrm{~kW}$ could then be transferred into $170 \mathrm{~mA}$ of beam.

In December 2002, the cavity module was removed from the ESRF storage ring as the space was required for a new beam line. Taking into account the results of the test, it is now planned to modify the existing prototype and to prepare it for the first commissioning phase of the SOLEIL storage ring starting in April 2005.

It is also planned to build a second unit, which is required and will be installed in the SOLEIL ring for a second phase in order to reach full performance of the machine in 2006: $500 \mathrm{~mA}$ at $2.75 \mathrm{GeV}$, the ring fully equipped with insertion devices.

\section{TEST OF THE HOM DAMPED SC SOLEIL CAVITY AT THE ESRF}

Figure 1 shows the SC HOM-free SOLEIL prototype module in the ESRF storage ring. The module, the test configuration at the ESRF and the cooling plant have already been described extensively $[1,2,3]$.

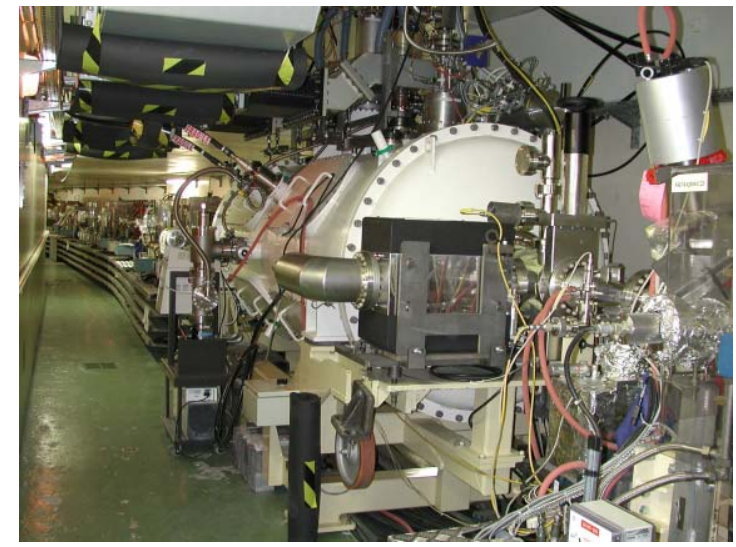

Figure 1: SOLEIL SC module in the ESRF storage ring 
The main design aspects that have been optimised for a reliable HOM-free operation in high intensity rings $[1,2$, 3] are briefly reminded below:

- On the $400 \mathrm{~mm}$ diameter beam tube connecting two cells in a single cryostat, the HOMs are effectively coupled with conventional coaxial couplers, which extract the power from the structure. As no ferrite absorbers are needed in the beam tubes, possible vacuum contamination is avoided.

- The open structure exhibits an excellent conductance for a very efficient vacuum pumping directly on the main tapers at each side.

- A high reliability is thus expected, which is essential for light sources with an expected MTBF better than 50 hours and more than $96 \%$ beam availability [4].

- The CERN technology using niobium coated copper cells has been adopted for the SOLEIL cavity. It is the first application to a cavity designed for high beam current.

The cooling circuit, the vacuum configuration as well as the connection to the $3^{\text {rd }}$ RF transmitter of the ESRF were thoroughly described in [3].

\section{ACHIEVEMENTS}

As already reported in [3], 5700 litres of liquid helium from three Dewars were sufficient to cool down and operate the SC module at $4 \mathrm{~K}$ for about 17 to 20 hours.

\section{Passive Operation at $300 \mathrm{~K}$}

During normal user operation of the storage ring, the SC module was kept at $300 \mathrm{~K}$ by means of a $15 \mathrm{~m}^{3} / \mathrm{h}$ flow of helium gas $(\mathrm{GHe})$ circulating through the cryostat. Less than $100 \mathrm{~W}$ of heat load from the beam had to be removed from the accelerating structure, naturally detuned by $1204 \mathrm{kHz}=3.4 f_{\text {revolution }}$ at $300 \mathrm{~K}$ [3]. This is slightly less than the worst case computations, which had predicted between 200 and $300 \mathrm{~W}$ of heat load.

A maximum HOM power of $50 \mathrm{~W}$ was measured on monopole HOM couplers in 16 bunch filling at $90 \mathrm{~mA}$ and no HOM driven coherent multibunch instability was ever observed up to the maximum ESRF intensity of $200 \mathrm{~mA}$. The system proved to be very reliable and transparent to machine operation, causing only three beam trips over a full year, which could not clearly be attributed to the cavity itself.

\section{Passive Operation at $4 \mathrm{~K}$}

In passive operation at $4 \mathrm{~K}$, as at $300 \mathrm{~K}$, the cavity remained transparent to the beam and no instability was detected up to $200 \mathrm{~mA}$ : along with the experience at $300 \mathrm{~K}$, this demonstrates the validity of the concept for an effective HOM damping.

\section{RF Voltage Conditioning at $4 \mathrm{~K}$}

The SOLEIL prototype module had already been powered in the CERN teststand prior to its installation at the ESRF, and it was relatively easy and fast to recondition the couplers up to the nominal total voltage of $4 \mathrm{MV}$ in $\mathrm{CW}$ and up to $5 \mathrm{MV}$ in pulsed mode.

After the second test window at $4 \mathrm{~K}$ in May 2002, a problem with the HOM coaxial feed-throughs was encountered, which limited the maximum voltage [3]. It turned out that the brazing joints close to the external RF connectors, between atmosphere and insulation vacuum, were leaking. As a consequence, some glow discharges occurred inside the coaxial lines and limited the voltage due to the spurious coupling to the fundamental mode. Once identified, the leaks were easily repaired. One of the coaxial lines showing strong traces of the discharges was cleaned up. At the August test, a decade was gained in the insulation vacuum and the accelerating voltage of the module could be raised to its nominal value.

The dipolar HOM couplers which are located close to the accelerating cells are equipped with notch filters for the rejection of the fundamental mode coupling. Due to an incorrect tuning of these filters and to the resulting spurious coupling to the fundamental mode, at $4 \mathrm{MV}$ in $\mathrm{CW}$, the extracted power was as high as $1650 \mathrm{~W}$. Moreover, the LHe circuits feeding the HOM couplers are not yet fully optimised and the cooling efficiency is only moderate. Altogether this lead to strong overheating of the HOM couplers at high accelerating voltage and was probably the reason for some quench-like events, with pressure bursts on the LHe tank.

At $4 \mathrm{MV}$, the total cryogenic losses were evaluated around $140 \mathrm{~W}$, which is slightly above the already high static losses of ca. $117 \mathrm{~W}$ measured in previous tests [3].

Some multipacting was also observed in the main RF couplers around $4 \mathrm{MV}$, eventually leading to some pressure bursts on which the RF was tripped. The fast vacuum interlock had been implemented to protect the ceramic against sputter deposition of copper. It is expected that further conditioning of the couplers will help to overcome multipacting.

\section{Beam Acceleration at $4 \mathrm{~K}$}

With $5 \mathrm{MeV}$ energy loss per turn at the ESRF, a minimum of $7 \mathrm{MV}$ of RF voltage is necessary for stable storage of a high intensity beam. At the end of the $4^{\text {th }}$ test window, in October 2002, $170 \mathrm{~mA}$ of beam could be stored, achieving 30 hours of lifetime with $3 \mathrm{MV}$ from the superconducting SOLEIL module and $4 \mathrm{MV}$ from the existing normal conducting ESRF cavities. This corresponds to $360 \mathrm{~kW}$ of power transferred from the SC cavity module into the beam and meets the requirements for the commissioning of the SOLEIL ring with one module. Surprisingly, only little time was needed for conditioning so that the beam intensity and the beam loading of the module could be ramped within a few hours.

A run test of one hour was carried out at the end of the last test period, with a smooth beam decay from 155 to $149 \mathrm{~mA}$, demonstrating that more than $300 \mathrm{~kW}$ can be fed to the beam over a longer period of time, without any thermal run away. 
REFURBISHMENT OF THE PROTOTYPE AND PRODUCTION OF A SECOND UNIT

Before its installation in the SOLEIL storage ring, taking into account the numerous results from the beam tests at the ESRF, the prototype module will be refurbished with the following major modifications:

- A copper shield cooled by liquid nitrogen will be inserted in order to reduce the static losses. Straps anchored on the shield will be installed to draw heat from the HOM couplers, the bulky tuning system, the coaxial lines, etc...

- On the dipole HOM couplers, the notch filter for the rejection of the fundamental mode coupling is tuned by adjusting the gap between the loop and the coupler walls, using a single wave bellow. Unfortunately, the bellow flexibility did not meet the initial specification and prevented from tuning the filter. The fabrication procedure is now being revised and the dipole HOM couplers will be replaced. This should allow to keep the power extracted from the fundamental mode at a reasonable level, even at a high accelerating voltage.

- Two superconducting modules will finally be installed to match the latest SOLEIL specifications for the revised nominal beam current, energy and losses from insertion devices. As a consequence, the nominal total accelerating voltage will be reduced from 4 to $3 \mathrm{MV}$ for phase I with only one module, and to $4.8 \mathrm{MV}$ for phase II with two accelerating modules in place. For power matching, a higher coupling to the cavity must therefore be set, with $Q_{\text {ext }}=110^{5}$ instead of $2.210^{5}$. To achieve this, the antennas of the fundamental power couplers will be cut, then lengthened, and the couplers re-conditioned to transfer up to $200 \mathrm{~kW}$ of $\mathrm{RF}$ power.

- In order to render the prototype operational for a light source, the instrumentation installed for the tests will be replaced with radiation-proof components. On this occasion, sensors with a wider temperature range will be used to follow cool-downs from room temperature to $4 \mathrm{~K}$.

- The helium circuitry will be modified to accommodate space for the thermal copper screen and to feed the HOM couplers from the bottom of the cryo-module. This should eliminate the experienced cooling problems.

These modifications will require an intervention in a clean area, followed by power tests. Helium processing is envisaged during the power tests. Modifications will be realised using the clean room and the power teststand at
CERN. A part of the intervention will also be performed at CEA-Saclay. For this purpose, a collaboration agreement between CERN, CEA and SOLEIL is being drawn up.

The intervention (dismounting, modifications, reassembling and tests) should be completed before October 2004, allowing the SOLEIL ring to be commissioned with the refurbished prototype in spring 2005.

For the fabrication of a second HOM-free cavity module, SOLEIL will issue calls for tender with the aim of placing orders beginning of 2004. The fabrication, assembly and tests should be completed by October 2005 . The installation and commissioning in the SOLEIL ring is scheduled at the end of 2005, allowing to reach full performance of SOLEIL in 2006.

\section{CONCLUSION}

The beam test of the strongly HOM damped superconducting SOLEIL cavity at the ESRF was extremely successful and brought all the expected results. The concept was validated, the voltage and power requirements of SOLEIL were reached. The tests also allowed the identification of a few design and fabrication weak points on the prototype, which can still be resolved before the installation of the module for the commissioning of the SOLEIL ring. With the planned modifications, the present performance limitations of the SOLEIL cavity are expected to be overcome. Based on these experimental results, the fabrication of a second module can now also be launched on a sound basis. Finally, the tests have also shown that this cavity constitutes a valuable option for a possible future intensity upgrade of the ESRF.

\section{ACKNOWLEDGEMENTS}

The contribution of many colleagues from CERN, CEA-DSM and the ESRF is greatly acknowledged. Special thanks are addressed to the CEA, CNRS and ILL in Grenoble for the liquid helium supply and the helium gas recuperation.

\section{REFERENCES}

[1] A. Mosnier et al., "Design of a heavily damped superconducting cavity for SOLEIL", PAC'97, Vancouver, June 1997.

[2] S.Chel et al., "Power Tests of the SOLEIL cryomodule", EPAC'00, Vienna, June 2000.

[3] J. Jacob et al., "Test of the SOLEIL Superconducting Cavity Proptotype on the ESRF Ring", EPAC'02, Paris, June 2002.

[4] J.-L. Revol et al. "Operation and Recent Developments at the ESRF", this conference. 